\title{
Safety criteria for flying E-sail through solar eclipse
}

\author{
Pekka Janhunen $^{\mathrm{a}, *}$, Petri Toivanen ${ }^{\mathrm{a}}$ \\ ${ }^{a}$ Finnish Meteorological Institute, Helsinki, Finland
}

\begin{abstract}
The electric solar wind sail (E-sail) propellantless propulsion device uses long, charged metallic tethers to tap momentum from the solar wind to produce spacecraft propulsion. If flying through planetary or moon eclipse, the long E-sail tethers can undergo significant thermal contraction and expansion. Rapid shortening of the tether increases its tension due to inertia of the tether and a Remote Unit that is located on the tether tip (a Remote Unit is part of typical E-sail designs). We analyse by numerical simulation the conditions under which eclipse induced stresses are safe for E-sail tethers. We calculate the closest safe approach distances for Earth, Moon, Venus, Mars, Jupiter, Ceres and an exemplary $300 \mathrm{~km}$ main belt asteroid Interamnia for circular, parabolic and hyperbolic orbits. We find that any kind of eclipsing is safe beyond approximately 2.5 au distance, but for terrestrial planets safety depends on the parameters of the orbit. For example, for Mars the safe distance with $20 \mathrm{~km}$ E-sail tether lies between Phobos and Deimos orbits.
\end{abstract}

Keywords: electric sail, mission design, eclipse

\section{Introduction}

The solar wind electric sail (E-sail) is a newly discovered way of propelling an interplanetary spacecraft by employing the thrust produced by the natural solar wind plasma stream [1, 2]. The solar wind dynamic pressure is tapped by long, thin, centrifugally stretched and positively charged tethers (Fig. 1). According to numerical estimations, the E-sail could produce 500 $\mathrm{nN} / \mathrm{m}$ thrust per unit length [3]. Thus an E-sail with $2000 \mathrm{~km}$ total tether

\footnotetext{
* Corresponding author

Email address: pekka.janhunen@fmi.fi (Pekka Janhunen)

URL: http://www.electric-sailing.fi (Pekka Janhunen)
} 
length (for example with 80 tethers $25 \mathrm{~km}$ long each) would produce $\sim 1 \mathrm{~N}$ of thrust at 1 au [4]. The thrust scales as $1 / r$ where $r$ is the solar distance [3]. The predicted thrust versus propulsion system mass ratio ( $1 \mathrm{~N}$ thrust at $1 \mathrm{au}$ and 100-200 kg mass) is high enough that it would enable a large class of previously unattainable missions in the solar system such as sending a $\sim 200 \mathrm{~kg}$ probe at more than $50 \mathrm{~km} / \mathrm{s}$ speed out of the solar system to make in situ measurements of interstellar space beyond the heliopause [5].

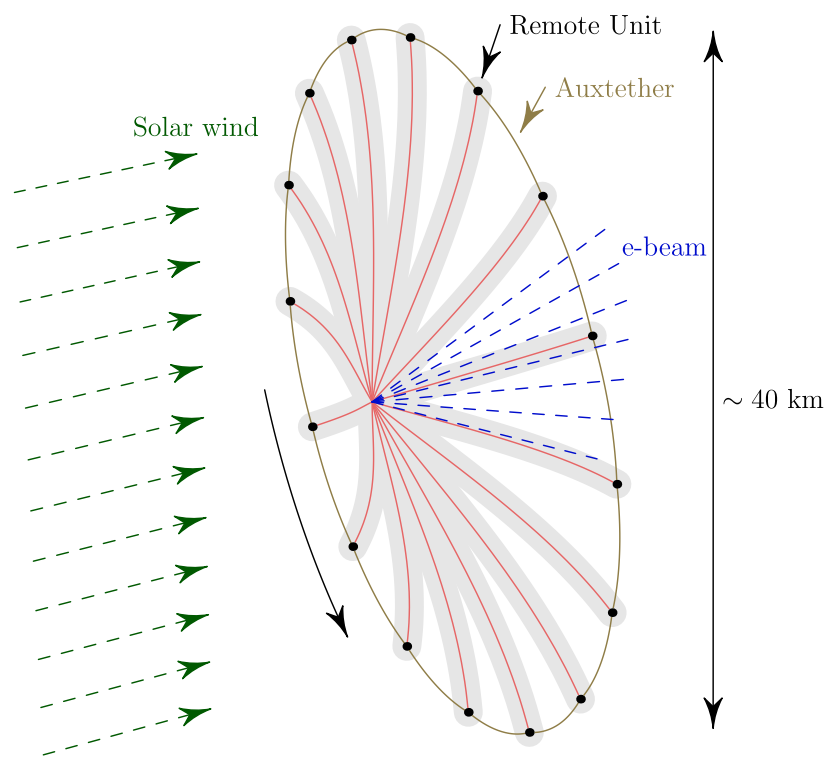

Figure 1: Spinning E-sail in the solar wind. The solar wind force bends the charged main tethers. The tethers are surrounded by the electron sheaths which are shown schematically by shading.

The metallic tethers of the E-sail (Fig. 2, [7, 8]) can be up to $20 \mathrm{~km}$ long. In theory and depending on the design, they might be even longer. If the Esail spacecraft enters into planetary eclipse, the tethers (mass per unit length of the four-wire aluminium tether is $11 \mathrm{~g} / \mathrm{km}$ ) cool down relatively rapidly because they are thin and thus have low heat capacity. Cooling causes the tethers to contract. Because the tethers are long, contraction corresponds to significant movement of the tether tip. Typically the tether tip contains a Remote Unit ( $\sim 0.5 \mathrm{~kg}$ mass) to which non-conducting auxiliary tethers are connected [4]. The contracting tether has to pull the masses residing at the tether tip inward, which requires certain force. This force causes first an increase of the tether tension and together with the centrifugal force 
afterwards also some oscillations. If the tension gets too high, the tether might break mechanically.

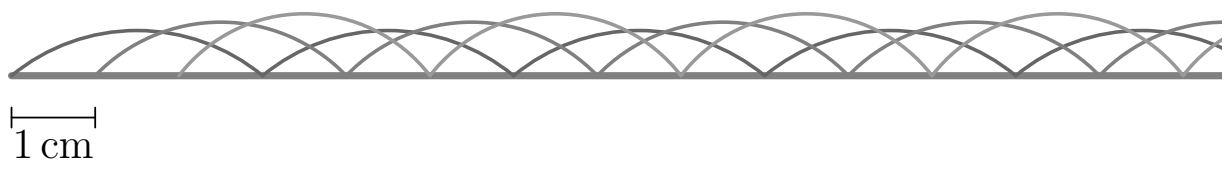

Figure 2: Micrometeoroid resistant four-wire E-sail Heytether.

Throughout the paper we assume $20 \mathrm{~km}$ tethers and use the value $1 \mathrm{cN}$ as the maximum allowed extra tension due to thermal contraction, which is $20 \%$ of the baseline overall tether tension of $5 \mathrm{cN}$ at $20 \mathrm{~km}$ tether length. If shorter tethers are used, risks due to thermal contraction are smaller.

The purpose of this paper is 1) to predict the eclipse induced extra tether tension for different solar system bodies and types of trajectories, and 2) to map the boundary between safe and unsafe parameter space valid for the baseline E-sail tether design. This information is needed when designing E-sail missions.

\section{Numerical model of tether eclipsing}

Consider a single E-sail tether which we assume to be a 4-wire Heytether [7] made of $r_{w}=25 \mu \mathrm{m}{ }^{1}$ radius aluminium base wire and three $25 \mu \mathrm{m}$ diameter loop wires. The load-bearing member is the base wire except at rare positions where micrometeoroids have broken the base wire. Hence we can ignore the elasticity of the loop wires in analysing eclipsing induced tensile stresses. We assume that there is an end mass $m$ at the tip of the tether which includes the mass of the Remote Unit and its share of the auxiliary tethers. Typically, $m=1 \mathrm{~kg}$ is clearly larger than the mass of the tether ( $\sim 0.2 \mathrm{~kg}$ at $20 \mathrm{~km}$ length), and we neglect the mass of the tether when solving the equation of motion of the tether. In thermal calculations the mass of the tether is essential and is included.

We denote the tether's rest length at its initial temperature $T_{0}$ by $L_{0}$. We assume a coefficient of linear thermal expansion $\alpha_{L}=2.31 \cdot 10^{-5} \mathrm{~K}^{-1}$ so that the tether's rest length at temperature $T$ is

$$
L=\left[1+\alpha_{L}\left(T-T_{0}\right)\right] L_{0} .
$$

\footnotetext{
${ }^{1}$ See Nomenclature at end of paper
} 
The tether aluminium base wire has Young elastic modulus $E=73 \mathrm{GPa}$, cross-sectional area $A=\pi r_{w}^{2}$ and spring constant

$$
k=\frac{E A}{L_{0}} .
$$

When put under tension $F$, the tether lengthens an amount $\Delta L$ such that Hooke's law holds:

$$
F= \begin{cases}k \Delta L, & \Delta L>0, \\ 0, & \text { otherwise }\end{cases}
$$

The tether base wire is heated by solar radiation whereas it is cooled by infrared emission. If the tether is perpendicular to sun direction, its thermal evolution is described by

$$
c_{p} \rho_{w} \pi r_{w}^{2} \frac{d T}{d t}=f \alpha 2 r_{w} I-2 \pi r_{w} \epsilon \sigma T^{4}
$$

where $c_{p}$ is the tether aluminium temperature-dependent heat capacity per unit mass, $\rho_{w}$ is the mass density $2700 \mathrm{~kg} / \mathrm{m}^{3}, r_{w}$ is the base wire radius $25 \mu \mathrm{m}, f$ is the non-eclipsed fraction of the Sun's limb, $\alpha=0.1$ is the optical absorptance of the tether, $I$ is the solar radiation power per unit area ( $1361 \mathrm{~W} / \mathrm{m}^{2}$ at $1 \mathrm{au}$ and scaling as $\sim 1 / r^{2}$ where $r$ is the solar distance), $\epsilon$ is the thermal emissivity (assumed to be 0.04 at $300 \mathrm{~K}$ and scaling linearly with $T$ ) and $\sigma=5.67 \cdot 10^{-8} \mathrm{~W} \mathrm{~m}^{-2} \mathrm{~K}^{-4}$ is Stefan-Boltzmann constant.

When the tether is not eclipsed, $f=1, d T / d t=0$ and $T=T_{0}$; hence

$$
\alpha I=\pi \epsilon \sigma T_{0}^{4} .
$$

Substituting Eq. (5) to Eq. (4) we obtain

$$
\frac{d T}{d t}=\frac{2 \epsilon \sigma}{c_{p} \rho_{w} r_{w}}\left(f T_{0}^{4}-T^{4}\right) .
$$

This equation allows us to compute the time development of the tether's temperature $T(t)$ when the eclipsing factor $f(t)$ is known from the orbit of the spacecraft. Eq. (1) can then be used to compute the tether's modified rest length $L(t)$. This quantity is plugged into our dynamical simulation which models the E-sail tether rig by solving Newton's laws of a set of point masses and their interaction forces. The tethers are modelled as massless force fields connecting the central spacecraft with Remote Units. In the baseline case we make the calculation with only one tether so that the dynamical model has three elements: spacecraft (a heavy point mass), Remote Unit (a lighter point mass) and a force field connecting them whose force law is Eq. (3). 


\section{Results}

Fig. 3 shows the time evolution of the tether's relative radiative heating $f$, temperature $T$ and tension $F$. The shown case is an exemplary event where the spacecraft is in circular orbit around Mars at the same radial distance as Deimos (6.92 Martian radii) and passes through the eclipse. The radiative heating $f$ is normalised so that $f=1$ corresponds to direct solar illumination. The small contribution from the planet's infrared emission is also included in $f$.
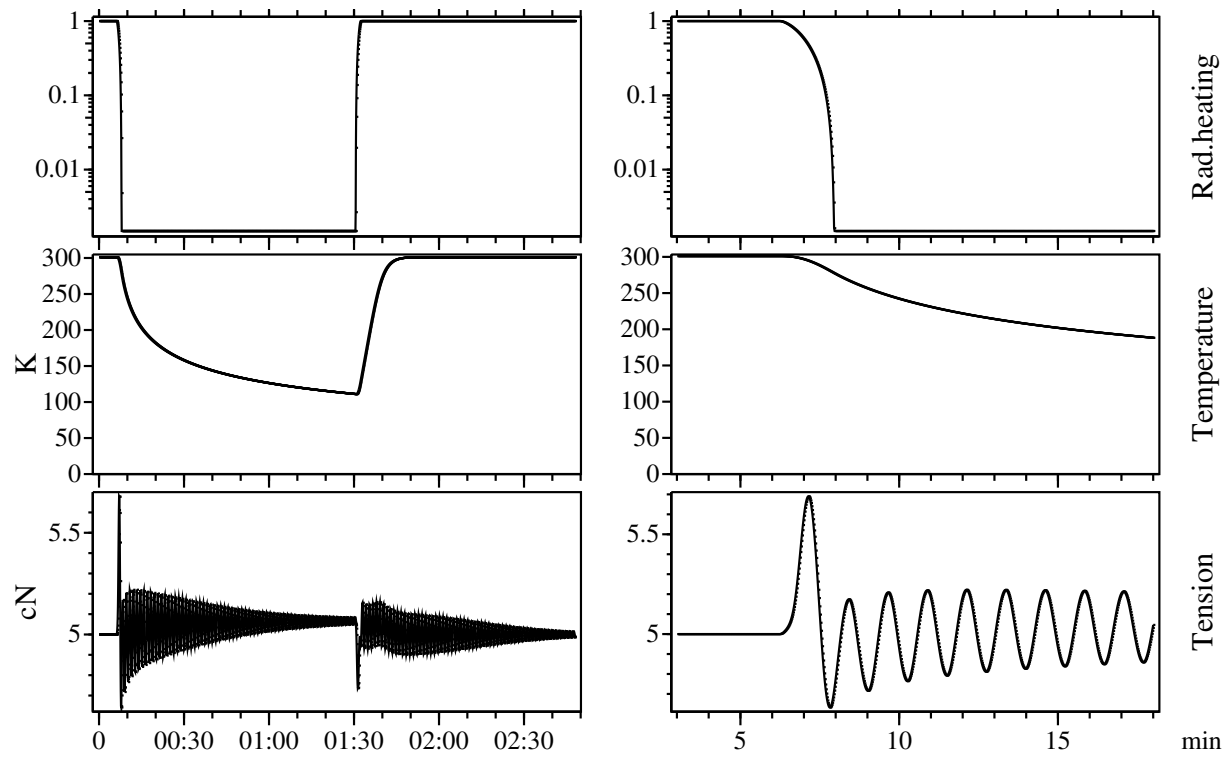

Figure 3: Relative radiative heating $f$, tether temperature $T$ and tether tension $F$ as function of time in circular orbit around Mars at Deimos distance (6.92 Martian radii). Right panel shows detail around the 10 min mark from the event's start).

The relative radiative heating drops from unity down to $\sim 10^{-3}$ (a value representing to Martian infrared heating) in about 2 minutes which is the duration of the penumbra phase in this case. The tether temperature drops slower, having dropped about $100 \mathrm{~K}$ during 10 minutes after the start of the eclipse. The eclipse lasts almost 1.5 hours and the minimum temperature reached is $110 \mathrm{~K}$. When the eclipse ends, the temperature was still decreasing slowly which implies that it had not yet reached complete radiative equilibrium. The tether's baseline tension was assumed to be $5 \mathrm{cN}$. The maximum 
Table 1: Properties of heavenly bodies.

\begin{tabular}{lll}
\hline Body & Solar distance & Mean radius \\
\hline Earth & $1 \mathrm{au}$ & $6371 \mathrm{~km}$ \\
Moon & $1 \mathrm{au}$ & $1737 \mathrm{~km}$ \\
Venus & $0.72 \mathrm{au}$ & $6052 \mathrm{~km}$ \\
Mars & $1.52 \mathrm{au}$ & $3390 \mathrm{~km}$ \\
Ceres & $2.77 \mathrm{au}$ & $476 \mathrm{~km}$ \\
Interamnia & $3.06 \mathrm{au}$ & $\sim 164 \mathrm{~km}$ \\
Jupiter & $5.2 \mathrm{au}$ & $69911 \mathrm{~km}$ \\
\hline
\end{tabular}

tension during the eclipse is $0.7 \mathrm{cN}$ larger. The thermal contraction of the tether produces an extra tension $-m \alpha_{L} L T^{\prime \prime}(t)$. The $25+50 \mu \mathrm{m}$ aluminium wire ultrasonic bonds (i.e. bonds between the $50 \mu \mathrm{m}$ base wire and $25 \mu \mathrm{m}$ loop wires) have a typical tensile strength of $10 \mathrm{cN}$. The $m=1 \mathrm{~kg}$ end mass of the tether oscillates with $74 \mathrm{~s}$ period which corresponds to the eigenfrequency $\omega=\sqrt{k / m}$ of the end mass when supported by the tether whose spring constant $k$ is given by Eq. (2).

To find out the maximum encountered extra tension, one has to make runs for different orbital parameters. Fig. 4 shows the result for Earth, Moon, Venus, Mars, Ceres, Interamnia (a $330 \mathrm{~km}$ diameter main belt asteroid) and Jupiter. Three types of orbits are shown: circular planetary orbit (solid line), parabolic zero total energy orbit (dashed line) and hyperbolic orbit which has speed $v_{\infty}=15 \mathrm{~km} / \mathrm{s}$ at infinity (dotted line). Basic parameters of the objects are listed in Table 1 .

Let us consider the solar system bodies appearing in Fig. 4 in turn. For Earth, circular and parabolic orbits are safe if the distance is larger than $\sim 8-10 R_{E}$. Hyperbolic orbits are unsafe unless the closest distance is beyond $20 R_{E}$. The differences between orbits are mainly due to their speed. The higher the speed, the faster is the eclipse's onset and termination, and faster changes in illumination cause faster changes in temperature and larger acceleration of the end mass which determines the extra tension that the tether has to withstand. The Moon case is similar to Earth. The only difference is that the duration of the eclipse's onset and termination is determined by the absolute distance to the eclipsing body and for example 10 Moon radii is a much shorter distance than 10 Earth radii.

Venus is more hostile than Earth, which is due to the closeness of the Sun and the associated larger thermal contrast between direct sunlight and 


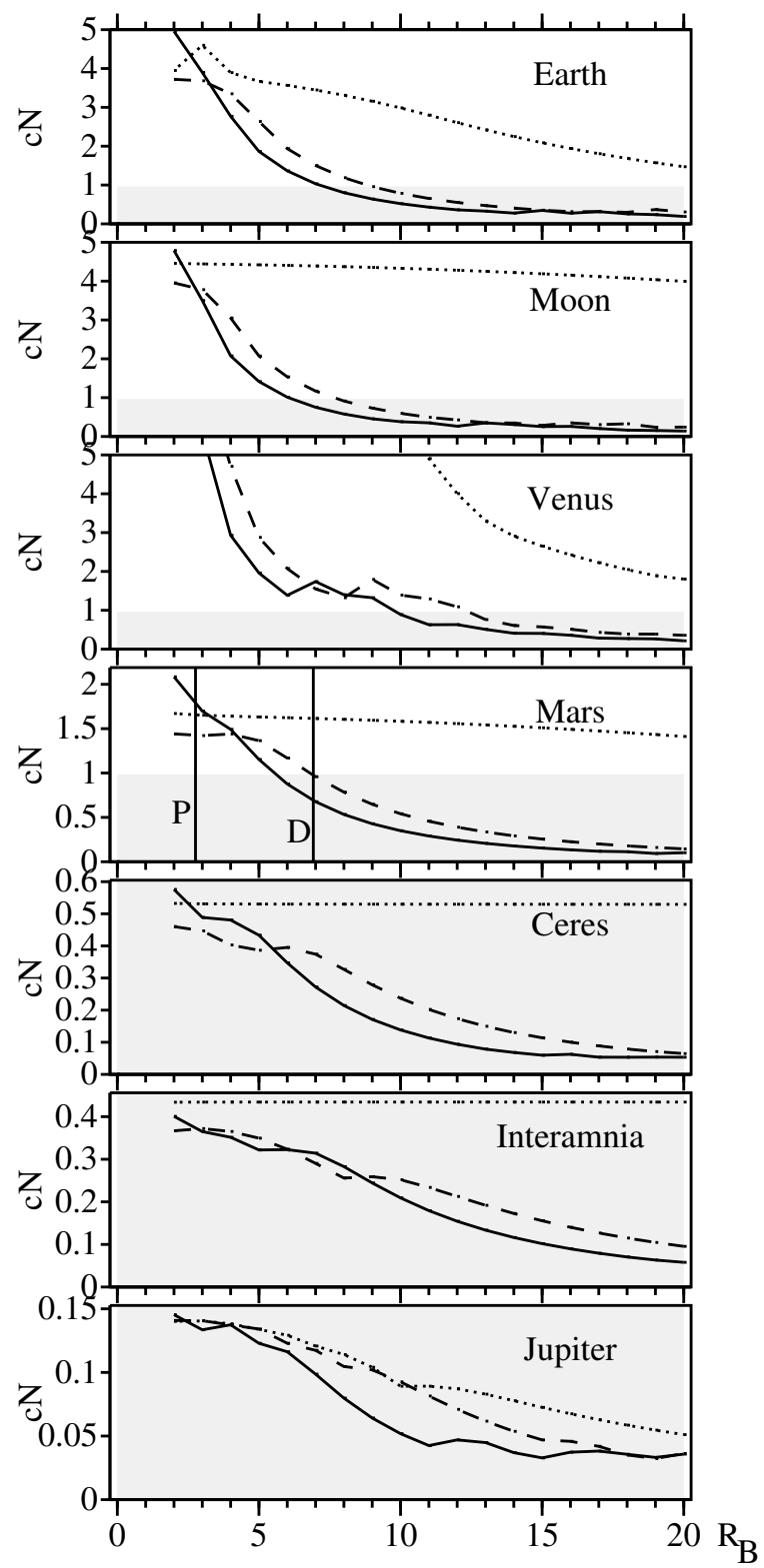

Figure 4: Maximum encountered extra tether tension for nightside passes through shadows of heavenly bodies. Horizontal axis is the closest distance from the body's centre in units of body radius. Solid line is circular orbit, dashed line parabolic orbit and dotted line hyperbolic orbit with $v_{\infty}=15 \mathrm{~km} / \mathrm{s}$. Shading denotes approximate safe region where extra tension is less than $1 \mathrm{cN}$. 
Table 2: Safe regions for eclipsed E-sails in different orbits. The numbers are in units of body radius. Largest permissible thermally induced tether tension of $1 \mathrm{cN}$ is assumed. The hyperbolic orbit has $v_{\infty}=15 \mathrm{~km} / \mathrm{s}$.

\begin{tabular}{llll}
\hline Body & Circular & Parabolic & Hyperbolic \\
\hline Earth & $\geq 7$ & $\geq 9$ & None \\
Moon & $\geq 6$ & $\geq 8$ & None \\
Venus & $\geq 10$ & $\geq 12$ & None \\
Mars & $\geq 5.5$ & $\geq 7$ & None \\
$\gtrsim 2.5$ au & Any & Any & Any \\
\hline
\end{tabular}

eclipse. Solar radiation flux scales as $1 / r^{2}$ where $r$ is the solar distance. Before eclipse the tether is in thermal equilibrium so the tether's temperature is such that its emitted infrared is equal to solar heating. Thus, when eclipsing starts, the initial cooling power also scales as $1 / r^{2}$ and the time derivative of the temperature is larger if the body is closer to the Sun. By the same logic, Mars should be more benign, and this is indeed the case in Fig. 4. The Mars panel also shows Phobos (P) and Deimos (D) orbital distances marked. Deimos is possible to reach by parabolic and circular orbit, but Phobos is not. Reaching Phobos by pure E-sail propulsion is by no means out of question, although it is a bit too risky for the baseline E-sail with $20 \mathrm{~km}$ tethers.

Both small and large outer solar system objects (main belt asteroid Interamnia, dwarf planet Ceres and Jupiter) seem safe to pass at any distance and for all considered orbits. In the outer solar system, the Sun's apparent diameter is smaller which makes the eclipse's onset and termination faster than in the inner solar system. By itself, this would increase the stress on the tether, but the cooler initial temperature of the tether reduces $d T / d t$ so significantly that this effect is masked out. For Jupiter there is not much difference between different types of orbit. Jupiter's gravity field is so strong that the speed difference between parabolic and $v_{\infty}=15 \mathrm{~km} / \mathrm{s}$ hyperbolic orbit is not dramatic.

Table 2 summarises the safe regions. Elliptical orbits fall in between the circular and parabolic ones.

\section{Discussion}

For E-sail mission analysis, the relevance of planetary orbits is the following. Pure E-sail propulsion enables rendezvous with a planet, for example 
Mars or Venus [6]. By rendezvous we mean that the spacecraft's heliocentric orbit coincides with the heliocentric orbit of the planet, so that in the planet's frame of reference the orbit is parabolic. It is possible to use E-sail propulsion to change the parabolic orbit into a bound elliptic or circular orbit. When doing so, the closest nightside approach distance of circular orbits is important to know. Of course, because E-sail does not produce propulsion inside magnetosphere, bound Earth orbits are not very interesting in the Esail context. Hyperbolic orbits are relevant if one wants to combine E-sail propulsion with traditional planetary gravity assist manoeuvres.

The baseline maximum usable tension of the baseline E-sail Heytether ( $50 \mu \mathrm{m}$ base wire and $25 \mu \mathrm{m}$ loop wires) is about $5 \mathrm{cN}$ which is about $50 \%$ of the mean tensile strength of the ultrasonic bonds between 25 and $50 \mu \mathrm{m}$ aluminium wires. From this, we estimated that the extra tension due to eclipsing can be $1 \mathrm{cN}$. This could be achieved either by increasing the safe load to $6 \mathrm{cN}$ by improved manufacturing technology or by improved qualification process, or by reducing the basic tension to $4 \mathrm{cN}$. With some temporary penalty in E-sail thrust, it would also be possible to reduce the spin rate temporarily for the duration of the eclipse.

The tension due to thermal contrasts scales linearly with the length of the tethers. If the tethers are shorter than our assumed $20 \mathrm{~km}$, the effects are correspondingly weaker. Also, making the tether wires thicker and reducing the mass of the Remote Unit would make the tethers more tolerable to thermal contrasts. With some modifications of this kind, reaching for example Phobos in rendezvous mode would very likely be feasible.

Redesigning the E-sail so that it could tolerate eclipsed gravity assist manoeuvres with Venus, Earth or Mars would be a tall order, because of the severity of the thermal stresses in that case. It might only be feasible by some rather radical change of the design philosophy of the E-sail tether. We remark, however, that this state of affairs is unlikely to be a problem in practice, because gravity assist manoeuvres are typically not needed in E-sail missions. Even if one wants to make use of them with terrestrial planets, it might be possible to design the flyby in such a way that eclipsing is avoided. In any case, as we pointed out above, eclipsing considerations do not seem to limit the use of gravity assist manoeuvres with Jupiter and other giant planets.

In the calculations presented, we considered only a single E-sail tether. To check the goodness of this assumption we also made runs with multitether E-sail geometries where the tips of the main tethers are connected by 
auxiliary tethers for dynamical stability. The results were very similar to the single-tether runs. Hence there is good reason to believe that the single tether results reported above are representative of real E-sails with a larger number of tethers.

\section{Conclusions}

We briefly summarise our main results:

1. Bound orbits around terrestrial planets and the Moon are conditionally E-sail safe depending on the distance of closest eclipsed approach and on the type of the orbit (Table 22). For example for Mars orbits, reaching Deimos in rendezvous mode with E-sail propulsion would be safe, while rendezvous with Phobos would likely require some modifications to the baseline E-sail.

2. For the terrestrial planets, eclipsed orbits with significant hyperbolic excess speed are unsafe. Thus, gravity assist manoeuvres with Venus, Earth and Mars are safe to perform with deployed E-sail only if the orbit is designed such that eclipsing does not occur.

3. Beyond $\sim 2.5$ au solar distance, any eclipsing is safe for the E-sail.

4. These conclusions hold for $20 \mathrm{~km}$ tethers. For shorter tether length the risks due to eclipsing are proportionally smaller.

\section{Acknowledgement}

We acknowledge the Academy of Finland (grant 250591) for financial support.

\section{References}

[1] P. Janhunen, Electric sail for spacecraft propulsion, Journal of Propulsion and Power 20 (4) (2004) 763-764.

[2] P. Janhunen, Electric sail for producing spacecraft propulsion, U.S. Pat. 7641151, Filed March 22006 (2010).

[3] P. Janhunen, Increased electric sail thrust through removal of trapped shielding electrons by orbit chaotisation due to spacecraft body, Annales Geophysicae 27 (2009) 3089-3100. 
[4] P. Janhunen, et al., Electric solar wind sail: towards test missions, Review of Scientific Instruments 81 (2010) 111301.

[5] P. Janhunen, P. Toivanen, J. Envall, S. Merikallio, G. Montesanti, J. Gonzalez del Amo, U. Kvell, M. Noorma, S. Lätt, Overview of electric solar wind sail applications, Proceedings of Estonian Academy of Sciences 63 (2014) 267-278.

[6] G. Mengali, A.A. Quarta, P. Janhunen, Electric sail perfromance analysis, Journal of Spacecraft and Rockets 45 (2008) 122-129.

[7] H. Seppänen, S. Kiprich, R. Kurppa, P. Janhunen, E. Hæggström, Wireto-wire bonding of $\mu \mathrm{m}$-diameter aluminum wires for the Electric Solar Wind Sail, Microelectronic Engineering 88 (2011) 3267-3269.

[8] Seppänen, H., T. Rauhala, S. Kiprich, J. Ukkonen, M. Simonsson, R. Kurppa, P. Janhunen, E. Hæggström, One kilometer (1 km) electric solar wind sail tether produced automatically, Review of Scientific Instruments 84 (2013) 095102. 


\section{Appendix A. Nomenclature}

A Cross-sectional area of tether base wire, $1.96 \cdot 10^{-9} \mathrm{~m}^{2}$

au Astronomical unit, $149597871 \mathrm{~km}$

$c_{p} \quad$ Heat capacity of tether material (aluminium), $910 \mathrm{~J} \mathrm{~kg}^{-1} \mathrm{~K}^{-1}$

E Young elastic modulus of tether material, $73 \mathrm{GPa}$

$f \quad$ Uneclipsed fraction of the solar limb

$F \quad$ Tether tension

I Solar radiative flux at spacecraft location (power per area)

$k \quad$ Spring constant of tether

$L \quad$ Tether length

$L_{0} \quad$ Rest length of tether at temperature $T_{0}$

$\Delta L$ Length change (elongation) of tether due to tension

$m \quad$ Tether end mass, $1 \mathrm{~kg}$

$r \quad$ Solar distance of eclipsing body

$R_{B} \quad$ Radius of eclipsing body

$R_{E} \quad$ Radius of Earth

$r_{w} \quad$ Radius of base wire of E-sail tether, $25 \mu \mathrm{m}$

$t$ Time

$T$ Tether temperature

$T_{0} \quad$ Tether initial temperature before eclipse

$v_{\infty} \quad$ Speed at infinity for hyperbolic orbit

$\alpha \quad$ Optical absorptance of tether surface, 0.1

$\alpha_{L} \quad$ Coefficient of linear thermal expansion of base wire, $2.31 \cdot 10^{-5} \mathrm{~K}^{-1}$

$\epsilon \quad$ Thermal infrared emissivity of tether surface, 0.04

$\rho_{w} \quad$ Mass density of tether material (aluminium), $2700 \mathrm{~kg} \mathrm{~m}^{-3}$

$\sigma \quad$ Stefan-Boltzmann constant, $5.67 \cdot 10^{-8} \mathrm{Wm}^{-2} \mathrm{~K}^{-4}$ 